\title{
ESTUDO DA VIABILIDADE DE USO DO LODO DE ESTAÇÃO DE TRATAMENTO DE ÁGUA COMO ADSORVENTE ALTERNATIVO
}

\author{
A de F. GOUVEIA ${ }^{1}$, C. KREUTZ ${ }^{1}$, K. Q. de CARVALHO ${ }^{2}$, F. H. PASSIG ${ }^{3}$, D. S. \\ $\mathrm{IKENO}^{4}$ e L. M. LUNARDI ${ }^{5}$.
}

\footnotetext{
${ }^{1}$ Universidade Tecnológica Federal do Paraná, Departamento Acadêmico de Ambiental

${ }^{2}$ Universidade Tecnológica Federal do Paraná, Departamento Acadêmico de Construção Civil

${ }^{3}$ Universidade Tecnológica Federal do Paraná, Departamento Acadêmico de Química e Biologia

${ }^{4}$ Universidade Tecnológica Federal do Paraná, Programa de Pós-Graduação em Engenharia Civil

${ }^{5}$ Universidade Tecnológica Federal do Paraná, Departamento Acadêmico de Engenharia Civil

E-mail para contato: gouveiaf.andreia@gmail.com
}

\begin{abstract}
RESUMO - Estima-se que $20 \%$ da produção mundial de corante usado na indústria têxtil sejam descartados no ambiente. Neste trabalho é avaliada a adsorção do vermelho remazol (VR) e azul de metileno (AM) com os materiais adsorventes: lodo de Estação de Tratamento de Água (LTA) e carvão ativado granular (CAG). Os resultados da caracterização físico-química do LTA e CAG foram $\mathrm{pH} 6,99$ e 6,24 ; densidade aparente 0,77 e $4,15 \mathrm{mg} / \mathrm{L}$; teor de umidade 2,45 e $10 \%$; teor de cinzas 12,82 e $3,50 \%$; teor de material volátil 15,98 e $8,00 \%$ e granulometria média de $0,75,5,0$ e $6,1 \mathrm{~mm}$, respectivamente. O comprimento de onda resultou em $450 \mathrm{~nm}$ para RB e $470 \mathrm{~nm}$ para AM. Os resultados das eficiências de remoção indicaram como melhor $\mathrm{pH}$ para $\mathrm{AM}$ de 9,5 e para VR de 5,5. A temperatura mais adequada foi de $60{ }^{\circ} \mathrm{C}$ e $30^{\circ} \mathrm{C}$, respectivamente. $\mathrm{O} \mathrm{CAG}$ resultou mais eficiente na remoção de ambos os corantes.
\end{abstract}

\section{INTRODUÇÃO}

A indústria têxtil é responsável pela geração de efluentes provenientes do uso de água e produtos químicos nas diversas etapas do processo. Esses resíduos, se não tratados corretamente, contribuem para degradação e contaminação do ambiente. Segundo Tomaz (2000), a indústria têxtil consome aproximadamente de 275 a $365 \mathrm{~L}$ de corante para tingir 1 $\mathrm{kg}$ de tecido na etapa do tingimento.

A composição desses efluentes é heterogênea, principalmente nas etapas de tingimento e acabamento, contendo material tóxico e recalcitrante, de difícil degradação. Calcula-se que no processo de tingimento das fibras têxteis, aproximadamente $20 \%$ da produção mundial de corante seja perdida para o ambiente (GUARATINI; ZANONI, 2000).

A dificuldade das indústrias têxteis em tratar seus efluentes é devido às características dos corantes que possuem baixa degradabilidade, o que acarreta na menor eficiência dos processos biológicos convencionais quando comparados aos processos físico-químicos. Usualmente a forma de tratamento utilizada para remoção do corante envolve processos 
físicos ou químicos, incluindo coagulação, floculação, oxidação avançada, $\mathrm{H}_{2} \mathrm{O}_{2} / \mathrm{UV}$, ozonização, troca iônica, irradiação, adsorção, dentre outros (VASQUES et al., 2011).

Dentro deste contexto, o objetivo deste trabalho é analisar a eficiência do lodo de Estação de Tratamento de Água (LTA) como material adsorvente de solução aquosa contendo vermelho remazol e azul de metileno, em substituição ao carvão ativado granular (CAG).

\section{MATERIAL E MÉTODOS}

\subsection{Preparação da Solução Aquosa}

Para preparação de cada solução aquosa utilizou-se $0,15 \mathrm{~g}$ de azul de metileno (Qhemis, $85 \%$ de conteúdo de corante, fórmula química $=\mathrm{C}_{16} \mathrm{H}_{18} \mathrm{~N}_{3} \mathrm{SCl}$; $\mathrm{FW}=319$; natureza $=$ azul básico) e $0,15 \mathrm{~g}$ vermelho remazol (corante azo, fórmula química $=\mathrm{C}_{25} \mathrm{H}_{15} \mathrm{~N}_{7} \mathrm{O}_{16} \mathrm{~S}_{5} \mathrm{Na}_{4} \mathrm{Cl}$; $\mathrm{FW}$ =984,82; natureza $=$ vermelho) dissolvidos em $1 \mathrm{~L}$ de água potável (de torneira) sem qualquer tratamento adicional. Os corantes foram testados separadamente.

\subsection{Material Adsorvente}

Para caracterização físico-química do lodo de ETA foram determinados pH (ASTM 3838-80/1999), densidade aparente (ABNT NBR 12076/1991), teor de umidade (ASTM D 2867-04/2004), teor de material volátil (ASTM D 5832-98/2003), teor de cinzas (ASTM D 2866-94/1999), número de iodo (ABNT NBR 12073/1991) e granulometria (ABNT NBR NM 248/2001) em triplicata para LTA e CAG (Tabela 1). A caracterização físico-química do carvão ativado granular foi realizada por Ikeno (2013) e os resultados foram utilizados para correlacionar com as características do lodo de ETA, visto que o carvão utilizado neste trabalho é o mesmo utilizado pelo referido autor.

\subsection{Ensaios de adsorção}

Os ensaios de adsorção foram realizados em triplicata com massas de 1,0 g do lodo de ETA e de carvão ativado granular adicionadas a $100 \mathrm{~mL}$ de solução de cada corante com concentração e $\mathrm{pH}$ conhecidos em erlenmeyers de $125 \mathrm{~mL}$. A variação do $\mathrm{pH}$ nos ensaios foi de 5,$5 ; 7,5 ; 9,5$ com $\mathrm{pH}$ inicial corrigido pela adição de soluções de $\mathrm{NaOH}(0,1 \mathrm{M})$ e $\mathrm{H}_{2} \mathrm{SO}_{4}$ $(0,1 \mathrm{M})$.

Os ensaios foram conduzidos em uma mesa agitadora com banho-maria Labor, modelo Banho Metabólico Dubnoff, sob agitação constante de $120 \mathrm{rpm}$ e temperatura de 30 e $60{ }^{\circ} \mathrm{C}$ durante $150 \mathrm{~min}$. Nesse período, alíquotas de $10 \mathrm{~mL}$ foram retiradas dos erlenmeyers nos intervalos de tempo de 15, 30, 60, 90, 120, 135 e $150 \mathrm{~min}$. O material adsorvente foi separado da solução em centrífuga Sislab, modelo Twister, sob agitação de $2500 \mathrm{rpm}$ durante 5 min.

A concentração residual foi determinada através de medidas de absorbância antes e após o tratamento com comprimento de onda definido por varredura no espectrofotometro Hach UV-vis, modelo DR 5000, com passo ótico de $1 \mathrm{~cm}$ em cubeta de quartzo.

\section{RESULTADOS E DISCUSSÃO}




\subsection{Caracterização físico-química do LTA e CAG}

Os resultados médios obtidos na caracterização físico-química do lodo de Estação de Tratamento de Água (LTA) e carvão ativado granular (CAG) são apresentados na Tabela 1.

Tabela 1 - Resultados médios da caracterização físico-química das amostras dos materiais adsorventes.

\begin{tabular}{c|c|c}
\hline PARÂMETROS & LTA & CAG \\
\hline $\mathrm{pH}$ & $6,99 \pm 0,10$ & $6,24 \pm 0,10$ \\
\hline Densidade Aparente $\left(\mathrm{g} / \mathrm{cm}^{3}\right)$ & $0,77 \pm 0,012$ & $4,15 \pm 0,21$ \\
\hline Teor de Umidade $(\%)$ & $2,45 \pm 0,47$ & $10,00 \pm 1,00$ \\
\hline Material Volátil $(\%)$ & $15,97 \pm 1,65$ & $8,00 \pm 1,00$ \\
\hline Teor de cinzas $(\%)$ & $12,78 \pm 0,24$ & $3,50 \pm 0,35$ \\
\hline Número de Iodo $\left(\mathrm{mgI}_{2} / \mathrm{g}\right)$ & $135,81 \pm 0,89$ & $650,00 \pm 3,06$ \\
\hline Granulometria $($ diâm. da partícula $-\mu \mathrm{m})$ & & \\
\hline$>500 \mu \mathrm{m}$ & $98 \%$ em massa & $96,84 \%$ em massa \\
\hline $300-150 \mu \mathrm{m}$ & $1 \%$ em massa & $3,04 \%$ em massa \\
\hline$<150 \mu \mathrm{m}$ & $1 \%$ em massa & $0,12 \%$ em massa \\
\hline Diâmetro médio $\left(\mathrm{d}_{10}, \mathrm{~d}_{50}\right.$ e $\left.\mathrm{d}_{60}\right)$ & 0,$75 ; 5,0$ e 6,1 & - \\
\hline Coeficiente de uniformidade & 8,13 & - \\
\hline
\end{tabular}

Os resultados de $\mathrm{pH}$ obtidos para $\mathrm{CAG}$ apresentam semelhança com àqueles verificados por Garg et al. (2004) na faixa de 6,5 a 7,5 e por Rangel et al. (2013) de 6,7. Para o LTA, os valores foram semelhantes ao encontrado por Kairanly (2011) de 7,2. A densidade aparente de $0,77 \mathrm{~g} / \mathrm{cm}^{3}$ foi superior a obtida por Loureiro (2012) de $0,62 \mathrm{~g} / \mathrm{cm}^{3}$ para LTA. Na determinação do teor de material volátil, a área superficial específica e distribuição de porosidade são afetados, sendo necessária atenção para que as temperaturas não acarretem em combustão do material (LOUREIRO, 2012).

O valor médio do teor de umidade foi de $2,45 \%$ para LTA e de $10,00 \%$ para CAG. É importante que as moléculas de água sejam removidas do sólido para que a atividade não seja reduzida. $\mathrm{O}$ valor encontrado para o $\mathrm{CAG}$ foi superior ao valor encontrado por Loureiro (2012) de 6,24\%. Os valores médios de teor de cinzas de 12,7\% para LTA e 3,5\% para CAG foram superiores a 2,5\% obtido por Garg et al. (2004).

O número de iodo de $650 \mathrm{mg} / \mathrm{g}$ para CAG foi superior ao obtido por Loureiro (2012) de $575,37 \mathrm{mg} / \mathrm{g}$. De acordo com a NBR 12073/1991 (ABNT, 1991), o limite mínimo de 600 $\mathrm{mg} / \mathrm{g}$ é recomendado, o que não foi observado para o LTA com $135,8 \mathrm{mg} / \mathrm{g}$. Na caracterização granulométrica foi possível observar que mais de 96\% dos materiais ficaram retidos em peneiras de diâmetro $>500 \mu \mathrm{m}$ para CAG e LTA. Este parâmetro é utilizado para análise e conhecimento das partículas dos materiais, uma vez que quanto menores forem, maior será a sua área superficial, facilitando a adsorção.

\subsection{Ensaios de Adsorção}

Com a varredura das soluções aquosas contendo os corantes, foi possível obter o comprimento de onda de $470 \mathrm{~nm}$ para o azul de metileno $\left(\mathrm{y}=0,1054+0,0175 ; \mathrm{R}^{2}=0,9947\right)$; 
e de $450 \mathrm{~nm}$ para o vermelho remazol $\left(\mathrm{y}=0,1+0,0292 ; \mathrm{R}^{2}=0,9902\right)$. Os resultados de adsorção obtidos com a variação do $\mathrm{pH} 5,5$ e da temperatura $\left(30{ }^{\circ} \mathrm{C}\right.$ e $\left.60{ }^{\circ} \mathrm{C}\right)$ para o LTA e CAG nas soluções aquosas são apresentados nas Figuras 1 e 2.

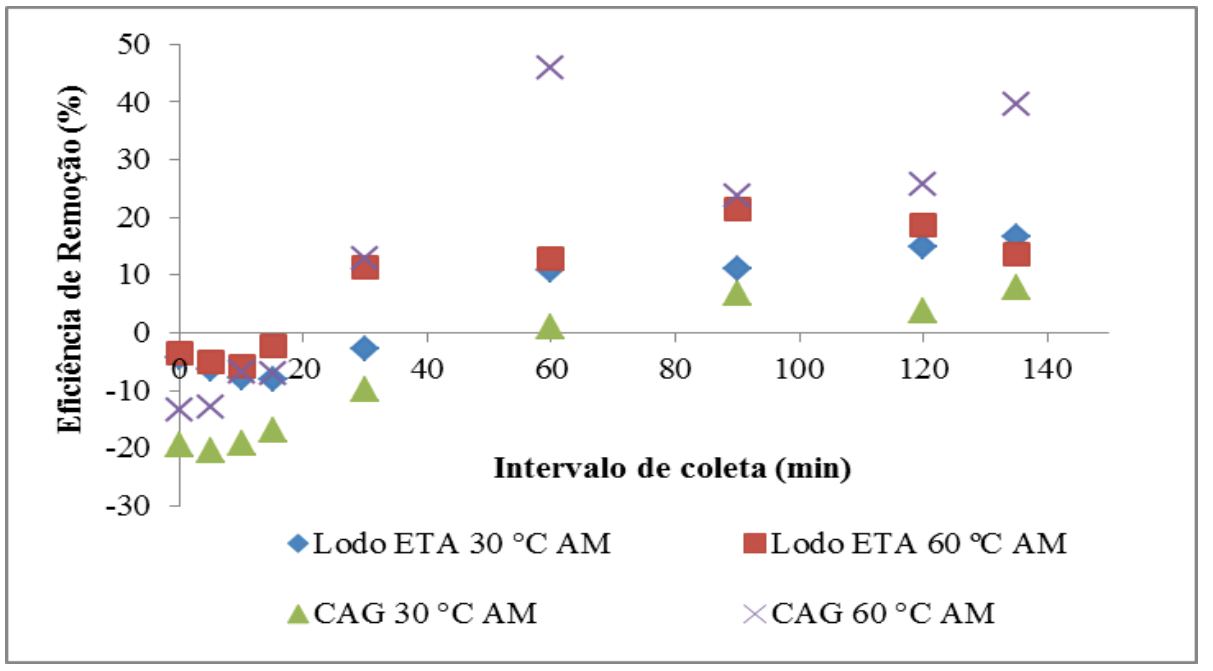

Figura 1 - Eficiência de remoção do corante AM para LTA e CAG pH 5,5 a $30^{\circ} \mathrm{C}$ e $60^{\circ} \mathrm{C}$.

Pela Figura 1 é possível notar melhor eficiência de remoção do azul de metileno com CAG quando comparada àquela verificada com o LTA. A melhor eficiência foi de $45,8 \%$ a $60{ }^{\circ} \mathrm{C}$ com pH 5,5 no tempo de $90 \mathrm{~min}$. Com o aumento da temperatura houve melhoria na eficiência de remoção do CAG de 7,7\% para 39,5\% no tempo de 150 min. O mesmo comportamento foi verificado para o LTA, pois a eficiência de remoção aumentou de $15,0 \%$ para $18,7 \%$ no tempo de $135 \mathrm{~min}$.

Foi possível verificar melhor eficiência de remoção do vermelho remazol com LTA em relação àquela obtida com o CAG (Figura 2). A melhor eficiência foi de $12,7 \%$ a $30^{\circ} \mathrm{C}$ com pH 5,5 no tempo de 90 min. Não foi verificada melhoria na eficiência de remoção para o LTA com o aumento da temperatura, pois esta reduziu de $6,6 \%$ para $4,1 \%$ no tempo de $150 \mathrm{~min}$. Com o aumento da temperatura foi verificado aumento na eficiência de remoção de cor com CAG de $0,5 \%$ para $6,5 \%$ no tempo de $150 \mathrm{~min}$.

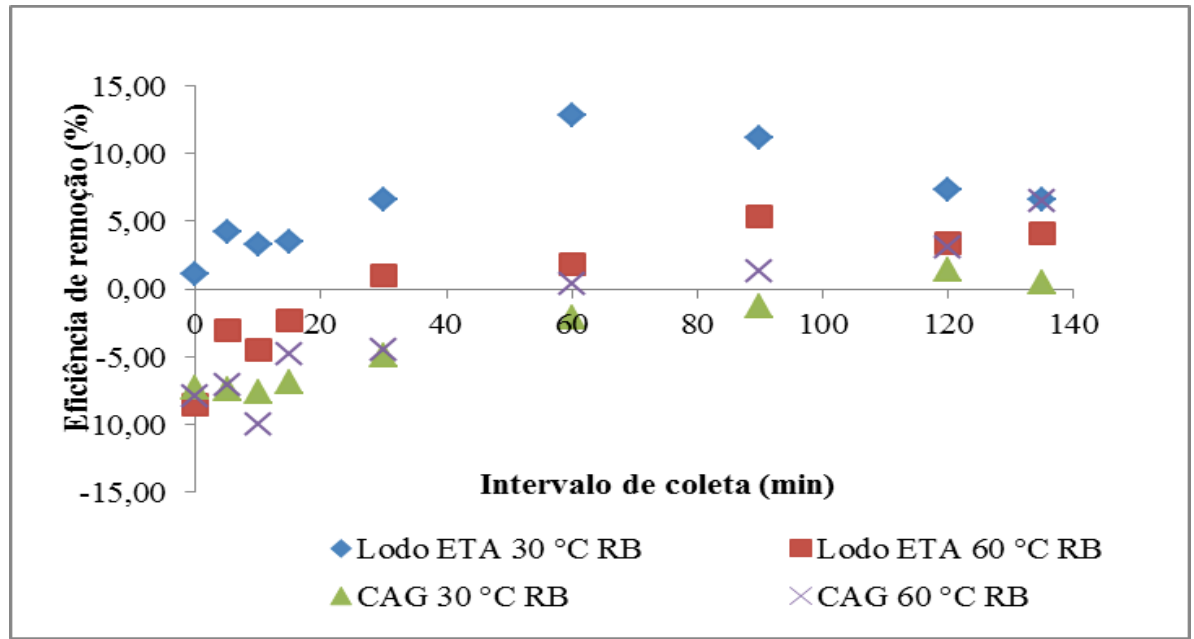

Figura 2 - Eficiência de remoção do corante VR para LTA e CAG pH 5,5 a $30^{\circ} \mathrm{C}$ e $60^{\circ} \mathrm{C}$. 
Os resultados de adsorção obtidos com a variação do $\mathrm{pH} 7,5$ e da temperatura $\left(30^{\circ} \mathrm{C}\right.$ e $60{ }^{\circ} \mathrm{C}$ ) para o LTA e CAG nas soluções aquosas contendo azul de metileno e vermelho remazol são apresentados nas Figuras 3 e 4.

Pela Figura 3 é possível notar que a eficiência de remoção do corante azul de metileno, em pH 7,5 foi maior para o lodo de ETA a $60{ }^{\circ} \mathrm{C}$ com remoção de $25,7 \%$ e para o vermelho Remazol foi de $5,0 \%$ para o CAG a $60{ }^{\circ} \mathrm{C}$. É possível observar que com o aumento da temperatura a remoção do azul de metileno aumentou gradativamente nos dois adsorventes testados, com melhores picos de adsorção na faixa de 90 a 120 min.

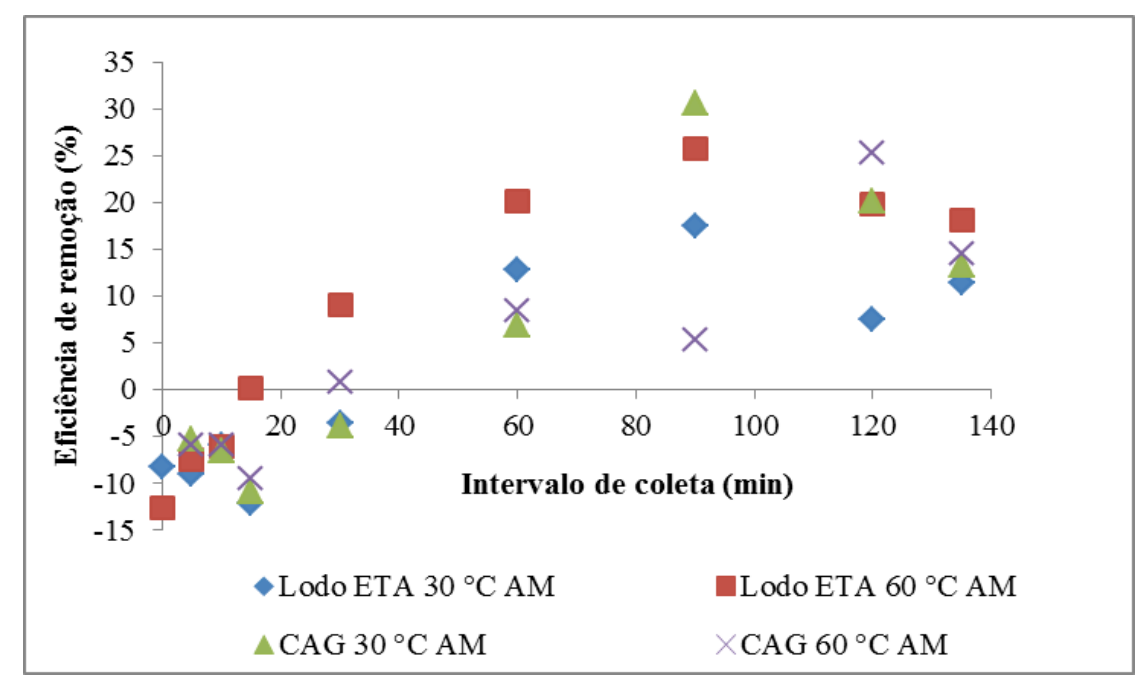

Figura 3 - Eficiência de remoção do corante AM para LTA e CAG pH 7,5 a $30^{\circ} \mathrm{C}$ e $60^{\circ} \mathrm{C}$

Pela Figura 4 é possível verificar que não foram obtidas eficiências de remoção significativas para o vermelho remazol, sendo o maior valor de 4,8\% pelo CAG em 120 min, e $2,7 \%$ pelo LTA em 5 min a $60{ }^{\circ} \mathrm{C}$.

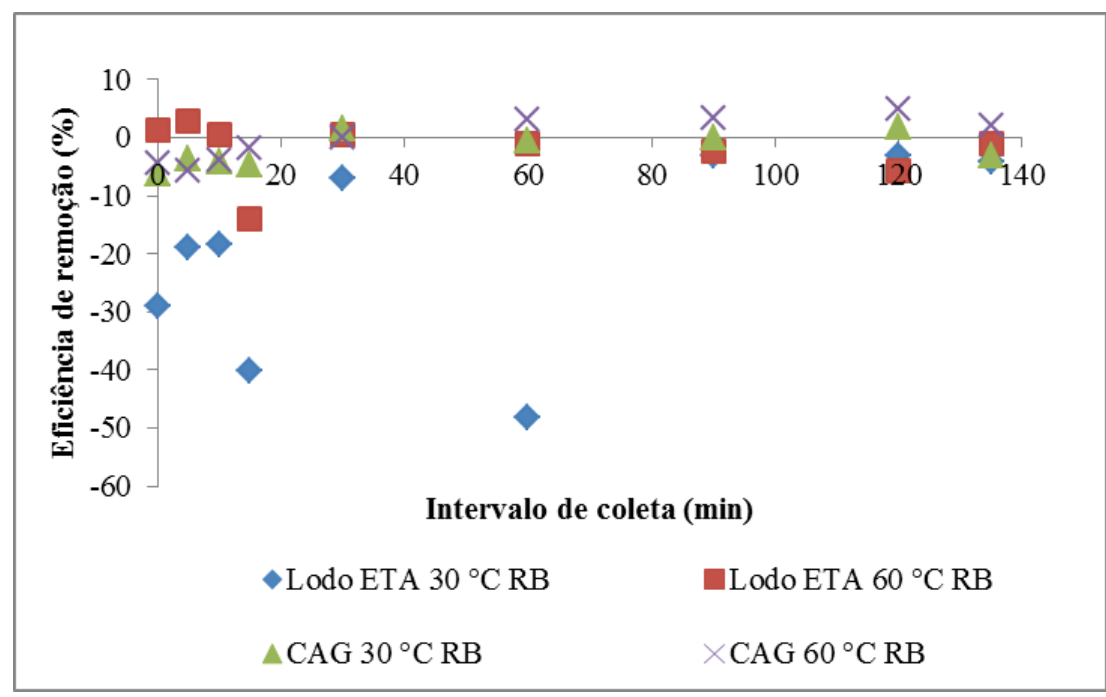

Figura 4 - Eficiência de remoção do corante VR para LTA e CAG pH 7,5 a $30^{\circ} \mathrm{C}$ e $60^{\circ} \mathrm{C}$. 
De acordo com os resultados é possível observar que em $\mathrm{pH} 7,5$, o VR é melhor adsorvido em temperaturas mais elevadas, haja visto que com temperatura de $30{ }^{\circ} \mathrm{C}$ a maior eficiência de remoção alcançada para o $\mathrm{CAG}$ foi de $1,8 \%$ e para LTA não houve adsorção. Os resultados de adsorção obtidos com a variação do $\mathrm{pH} 9,5$ a $30{ }^{\circ} \mathrm{C}$ e $60{ }^{\circ} \mathrm{C}$ para o LTA e CAG nas soluções aquosas são apresentados nas Figuras 5 e 6.

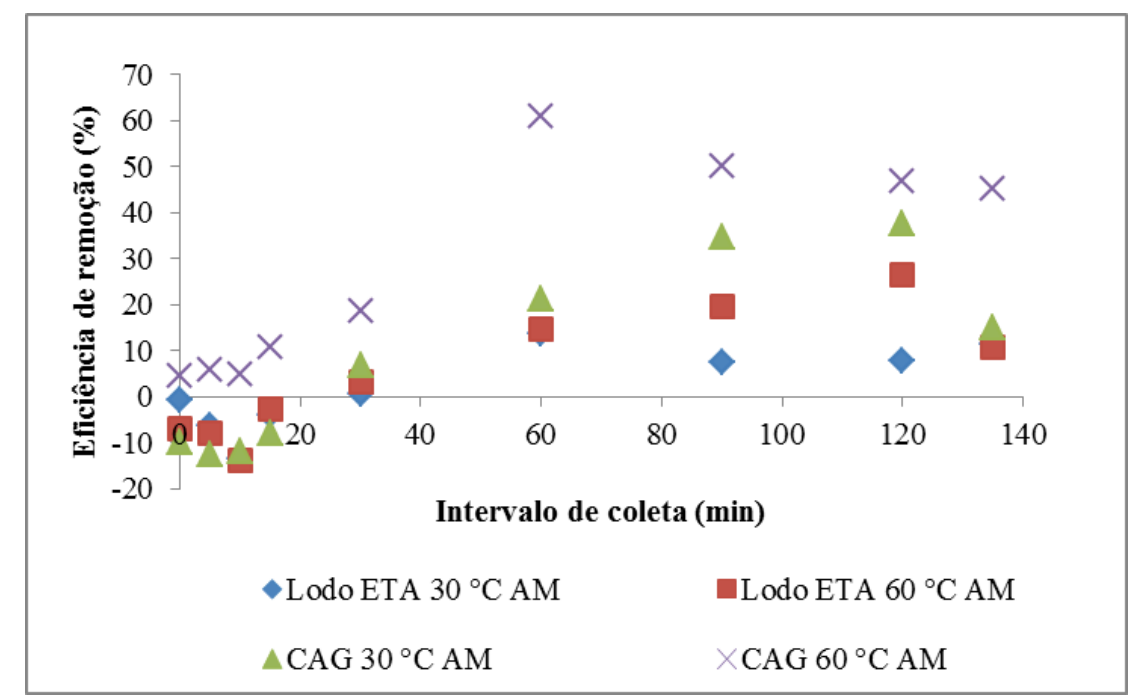

Figura 5 - Eficiência de remoção do corante AM para LTA e CAG pH 9,5 a $30^{\circ} \mathrm{C}$ e $60^{\circ} \mathrm{C}$.

Pela Figura 5 é possível notar que a eficiência de remoção do AM, em pH 9,5 foi maior utilizando o adsorvente $\mathrm{CAG}$ a $60{ }^{\circ} \mathrm{C}$, com $61 \%$ de remoção do corante. O CAG também foi mais eficiente adsorvente do corante VR para o mesmo pH a $30{ }^{\circ} \mathrm{C}$ com remoção de $6,6 \%$. Foi possível observar picos de adsorção no tempo de 120 min para CAG a $30{ }^{\circ} \mathrm{C}$ e LTA a $60{ }^{\circ} \mathrm{C}$ em 60 min para LTA a $30^{\circ} \mathrm{C} \mathrm{CAG} \mathrm{a} 60^{\circ} \mathrm{C}$.

Pela Figura 6 é possível notar que a eficiência de remoção do corante VR resultou constante para o CAG com remoção de $7,7 \%$ a $60{ }^{\circ} \mathrm{C}$ e $6,5 \%$ a $30^{\circ} \mathrm{C}$.

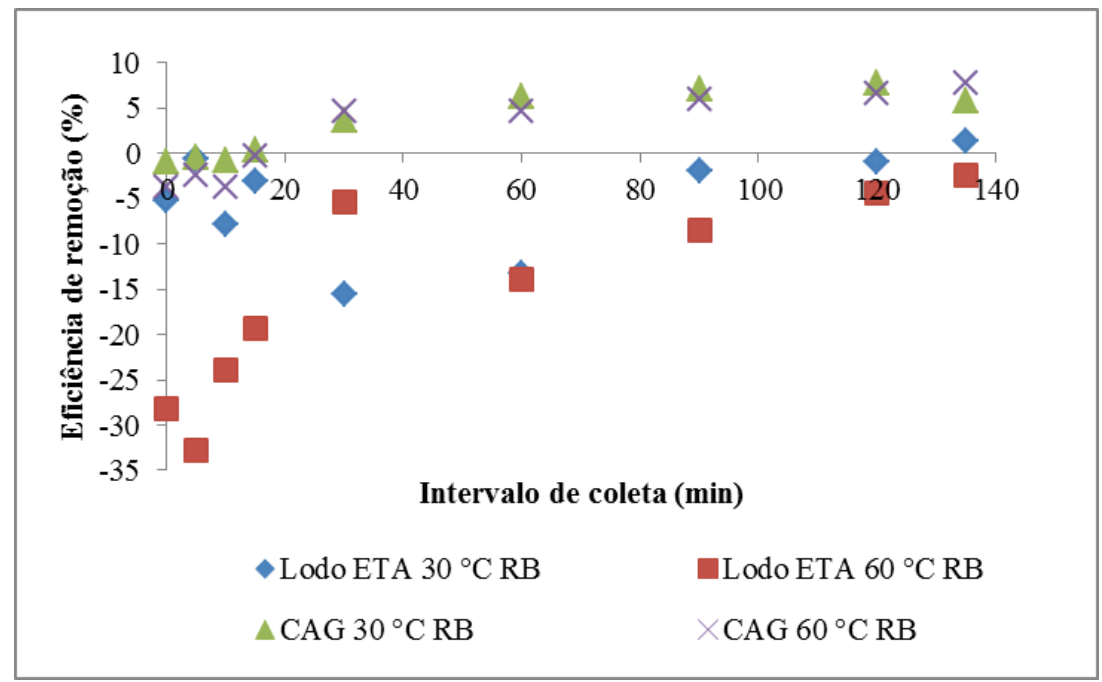

Figura 6 - Eficiência de remoção do corante RB para LTA e CAG pH 9,5 a $30^{\circ} \mathrm{C}$ e $60{ }^{\circ} \mathrm{C}$. 
Para o LTA, a eficiência máxima de adsorção registrada foi de $1,3 \%$ a $30{ }^{\circ} \mathrm{C}$ em 135 min. Não foi verificada melhoria deste percentual com aumento da temperatura para o LTA.

\section{CONCLUSÕES}

De acordo com os resultados experimentais, conclui-se que o LTA apresenta baixa eficiência de remoção para ambos os corantes testados. Este fato pode ser decorrente das propriedades orgânicas da amostra, ou seja, o LTA antes de adsorver os corantes, libera quantidade de matéria orgânica, contribuindo com o aumento da concentração de cor e turbidez da solução. Estudos mais aprofundados devem ser executados, alterando algumas propriedades físico-químicas do LTA, na tentativa de torná-lo um material adsorvente mais eficiente e financeiramente vantajoso quando comparado ao carvão ativado granular.

\section{REFERENCIAS}

ABNT - Associação Brasileira de Normas Técnicas. NBR 11834: Carvão Ativado Pulverizado - Especificação. Código Secundário: EB 2133. Rio de Janeiro, 1991.

NBR 12073: Carvão Ativado Pulverizado - Determinação de Iodo. Código Secundário: MB 3410. Rio de Janeiro, 1991.

NBR 12076: Carvão Ativado Pulverizado - Determinação da Massa Específica Aparente. Código Secundário: MB 3413. Rio de Janeiro, 1991.

ABNT - Associação Brasileira de Normas Técnicas. NBR NM 248: Agregados Determinação da composição granulométrica. Rio de Janeiro, 2003.

ASTM - American Society for Testing and Materials. D: 2867-2004: Standard Test Method for Moisture in Activated Carbon. 2004.

D: 2866-1994: Standard Test Method for Ash Content of Activated Carbon. 1999.

D: 5832-1998: Standard Test Method for Volatile Matter Content of Activated Carbon. 2003.

GARG, Vinod K.; AMITA, Moiranghthem.; KUMAR, Rakesh.; GUPTA, Renuka. Basic Dye (Methylene Blue) Removal From Simulated Wastewater By Adsorption Usin Indian Rosewood Sawdust: A Timber Industry. Hisar. Dyes and pigments, v. 63, n. 2004, p. 243250, nov. 2004.

GUARATINI, Claudia C. I.; ZANONI, Maria Valnice B. Corantes Têxteis. Araraquara. Química Nova, v. 23, n. 1, p 71-78, mar. 1999.

IKENO, Daniel S. Remoção de azul de metileno por adsorção usando pó de serragem do gênero Pinus sp. 64 f. Monografia (Trabalho de Conclusão de Curso) - Universidade Tecnológica Federal do Paraná, Campo Mourão, 64 p. 2013. 
KAYRANLI, Birol. Adsorption of Textile Dyes Onto Inron Based Waterworks Sludge From Aqueous Solution; Isotherm, Kinetic and Thermodynamic Study. Ankara. Chemichal Engineering Journal. v. 173 n. 2011. p 782-791, jun. 2011.

LOUREIRO, L. F. Avaliação do herbicida 2,4D em carvão ativado em pó e granular por meio de análise de isotermas de adsorção utilizando diferentes qualidades de água. 120 p. Dissertação (Mestrado em Engenharia Ambiental) - Universidade Federal do Espírito Santo, 2012.

RANGEL, Felipe N. et al. Caracterização físico-química do carvão ativado e do pó de serragem para adsorção. Universidade Tecnológica Federal do Paraná - UTFPR. Curitiba, 2013.

TOMAZ, Plínio. Previsão de Consumo de Água. São Paulo. Hermano \& Bugelli. Ed. Navegantes, 250 f. 2000.

VASQUES, Andressa R. et al. Adsorção dos corantes RO16, RR2 e RR141 utilizando lodo residual da indústria têxtil. Engenharia Sanitária e Ambiental, Florianópolis, v.16, n. 3, p. $1-8,2011$. 